offers an analysis of perceptions of community and conflict in parishes and medicalized neighbourhoods, while the sixth analyzes Shirley's The Lady of Pleasure, Brome's Covent Garden Weeded, and Nabbe's Covent Garden, as well as the so-called "running masques" of The Strand's grand households, in order to indicate the agency of theatre in creating perceptions of London's emergent West End.

The variety of texts investigated and the multiplicity of perspectives brought to bear on those texts sustain a sense of critical vitality throughout the book. But one of the costs of the proliferation of subsections is that there is little pressure to analyze literary passages in detail. Moreover, some of the book's key concepts, such as "spatial practice," tend to remain hazy. While parts of The Cultural Geography of Early Modern Drama will be edifying for professional literary scholars, its argumentative structure is unnecessarily diffuse, and it offers neither a model of sustained investigative focus nor sufficient account of what might be intended as decentring critical practice.

GLENN CLARK, University of Manitoba

Secchi Tarugi, Luisa (éd.).

Oriente e Occidente nel Rinascimento. Atti del XIX Convegno Internazionale (Chianciano-Pienza, 16-19 luglio 2007).

Florence: Franco Cesati Editore, 2009. 929 p. ISBN 978-88-7667-374-0 (broché) $125 €$

Le XXIV colloque de Chianciano-Pienza a eu lieu cette année. Il portait sur les rapports entre la Rome païenne et la Rome chrétienne à l'époque de la Renaissance. Comme d'habitude, il formera un beau volume qui sortira des presses précisément dans deux ans. Il faut saluer la régularité, la persévérance et la qualité du travail de Madame Luisa Secchi Tarugi, présidente de l'Istituto di Studi Umanistici Francesco Petrarca. Malgré les difficultés qui ne cessent d'augmenter au fil des années, cette institution fêtera bientôt son quart de siècle. C'est, pour tous ceux qui s'intéressent à la Renaissance, le dernier lieu annuel de réunion transdisciplinaire, un véritable colloque où, pendant trois jours (il a fallu malheureusement supprimer désormais une journée sur les quatre qui 
ont été longtemps la règle), il est possible de parler, avec des collègues venus de divers pays et spécialistes de sujets très différents, de ce qui a pu être un peu délaissé ces dernières années et qui, pourtant, importe vraiment : l'histoire des idées, pour employer une expression certes convenue mais parfaitement juste. C'est dire l'importance de cette institution discrète, en marge des circuits universitaires, comme jadis Pontigny puis Cerisy. Souhaitons que de telles rencontres ne disparaissent pas dans les prochaines années.

Ce volume, qui porte donc sur les rapports de l'Orient et de l'Occident, comprend soixante-six contributions (51 en italien, 9 en français, 4 en espagnol, 2 en anglais) qui abordent ce sujet sous les aspects les plus divers (principalement philosophiques, religieux, politiques, littéraires et artistiques). Un index de quarante pages complète un volume illustré, remarquable tant par sa qualité scientifique que par son élégance typographique. Il serait fastidieux de traiter ici de chacun des articles. On ne trouvera donc que les orientations principales.

Le livre s'ouvre par une étude sur les limites orientales assignées à l'Europe par les géographes de la Renaissance (M. Mund-Dopchie) ; un article sur la géographie chrétienne et la géographie musulmane à Florence sous Laurent le Magnifique (E. Haywood) suivi d'un autre sur les cartes géographiques vénitiennes du XVII ${ }^{e}$ siècle ( $\mathrm{S}$. Fabrizio-Costa) apporte au lecteur un cadre à la fois général et précis, tout comme les remarques sur les rapports linguistiques avec Constantinople (J.-L. Charlet). Les questions politiques ne sont pas oubliées; sont examinés entre autres les relations entre les Turcs et l'Espagne de Charles Quint et de Philippe II (J. Benavent), les rapports de l'Occident avec l'Afrique du Nord (D. Maffi), de l'Orient avec Venise (A. L. Puliafito), et l'importance de Lépante vue comme une nouvelle bataille de Salamine (A. Alonso). Cervantès et les barbaresques (L. Gentilli), le rôle commercial du golfe Persique (V. Fiorani Piacentini) et, enfin, la présence des Génois à Trébizonde (L. Radif) font également l'objet d'études. Le droit est abordé par une analyse d'une sentence de Botero sur le mariage en Turquie de marchands chrétiens (F. Buzzi).

La religion constitue le thème central de quelques communications portant sur les discours de Bessarion contre les Turcs (M. Lentzen), un appel à la Croisade sous le roi de France Charles VIII (G. Matteo Roccati), un catéchisme pour les Morisques (I. Colón), la comparaison des saints russes à saint François d'Assise (A. V. Romancuk), ainsi que le témoignage, au XVII siècle, du jésuite 
Roberto De Nobili (R. Osculati) envoyé aux Indes. Deux travaux portent sur le Japon : le premier sur un catéchisme portugais (M. Kubo), et le deuxième sur les premiers martyrs (A. Bresadola).

Les questions théologiques et philosophiques font l'objet de plusieurs contributions traitant de Leon Battista Alberti et le concile de Florence (A. Piccardi), des manuscrits de saint Jean Chrysostome à Florence (G. Masi), de la conception aristotélicienne et de la théologie chrétienne à propos de l'âme (A. Ghisalberti), de Giovanni Nesi pris entre prisca theologia et christiana religio (E. Tortelli), du codex de la Bibliothèque Laurentienne intitulé Comparatio philosophorum Aristotelis et Platonis (S. Ducic-Collette), des rapports de ces deux philosophes dans la pensée d'Alessandro Piccolomini (D. Costa), de l'intellect agent chez Alessandro Achillini et Pietro Pomponazzi (S. Benassi), de l'influence de la théologie de Denys l'Aréopagite sur la philosophie de Nicolas de Cuse (M. Laffranchi), de l'augustinien Andrea Biglia et de ses Commentarii historici de detrimento fidei in Oriente (T. Foffano), de l'Orient vu comme paradigme politique chez Étienne de La Boétie (A. Tarrête), et enfin de l'influence de l'Orient sur la magie naturelle (B. Lavillatte).

Le colloque n'a pas oublié les voyageurs et les savants. S. Chaudhuri, qui présente également un article sur les rapports de l'humanisme et de l'orientalisme au XVIII ${ }^{\mathrm{e}}$ siècle anglais, est ici l'auteur d'une étude su Nicolò de' Conti. On trouve aussi un article sur Pierre Belon (Y. Bellenger), dont traite également, en lui adjoignant Thevet et Nicolay, E. Mazeaud-Karagiannis. G. Mazzocchi s'intéresse au voyageur portugais Fernão Mendes Pinto, tandis que S. Di Francesco s'occupe de Batory, de Possevin et de la Moscovie.

Assurément, ce sont les relations des lettrés occidentaux et des Turcs qui comprennent le plus grand nombre d'études à propos de Plutarque. Elles portent sur Plutarque, tout d'abord (J. Spicka), Biondo Flavio (D. Defilippis puis S. Pittaluga et enfin G. Rossi), Antonio Galateo (I. Nuovo), Paolo Giovio (et Andrea Cambini ainsi que Marino Barlezio, par L. Michelacci), Cristoforo Buondelmonti (G. Ligato), Gianmaria Filelfo (F. Cairns), Érasme (É. Wolff), Juan Luis Vivès et Nicolas Clénard (G. Tournoy et G. Tournoy-Thoen), Miguel de Luna (E. Maggi), Francisco Agustín Tárrega (P. Pintacuda), et Luis de Góngora (R. Bonilla Cerezo). Deux imprimeurs sont évoqués : Oporin, qui exerce à Bâle (P. Bietenholz), et G. Ruscelli, à Venise (A. Maranini). A. Tarabochia Canavero s'occupe des emblèmes de Cesare Ripa. 
La littérature est précisément abordée dans huit études, dont les thèmes sont les suivants : une source de Béroalde de Verville, Cristoforo Armeno (D. Mauri), l'Orient chez Montaigne (J.-C. Ternaux), le Liber Proverbiorum de Lorenzo Lippi (P. Rondinelli), le Morgante de Pulci (M. Ballarini), les Octavas de Francisco de Aldana (V. Nardoni), Fernando de Herrera (G. Chiappini), la lyrique amoureuse de la fin du XVI ${ }^{e}$-début du XVII ${ }^{e}$ siècle (A. Colombo), ainsi que la perception de l'Orient dans la littérature hongroise (A. Di Francesco).

Enfin, l'art n'est pas oublié puisque sont examinés Piero della Francesca (A. Ceccarelli Pellegrino), l'impact des arts de l'Islam sur l'Europe (A. M. Martelli puis F. F. d'Arcais), l'architecture (L. Patetta puis M. Cerasi), et les rapports entre l'architecture et l'art pour la représentation de la cité orientale à la Scuola Grande di San Marco (T. Patetta).

On trouvera donc dans ce volume des études très précises et très fouillées (les notes abondantes sont une véritable mine d'informations), dont la diversité n'est pas un obstacle à la lecture, bien au contraire. Elle oblige le lecteur moderne à sortir de sa spécialité, à accéder à un univers culturel complexe dont il a bien souvent perdu non seulement les clefs mais même les éléments principaux.

François roudaut, Université Paul Valéry III, Montpellier

\section{Terjanian, Pierre.}

Princely Armor in the Age of Dürer: A Renaissance Masterpiece in the Philadelphia Museum of Art.

New Haven: Philadelphia Museum of Art in Association with Yale University Press, 2011. Pp. 54. ISBN 978-0-300-17631-5 (paperback) \$18.

In 2009 the Philadelphia Museum of Art acquired an exquisite horse armour originally commissioned in 1507 by the flamboyant German prince, Duke Ulrich of Württemberg. The decorative plate armour - a stunning product of the age of Dürer - was the work of the highly respected Nuremberg armourer Wilhelm Von Worms the Elder and is considered "to be among the finest examples of European armor anywhere" (5). Pierre Terjanian, the museum's associate curator of Arms and Armour, has produced a beautifully illustrated catalogue to mark the acquisition and public unveiling of the horse armour and 